\title{
Análise de trilha da contaminação por aflatoxinas em grãos de milho armazenados
}

\author{
Adriana Sbardelotto Di Domenico(1), Moeses Andrigo Danner ${ }^{(1)}$, Cleverson Busso(1), \\ Divair Christ( ${ }^{(2)}$ e Sílvia Renata Machado Coelho(2)
}

\begin{abstract}
(1)Universidade Tecnológica Federal do Paraná, Campus Dois Vizinhos, Estrada para Boa Esperança, Km 04, Comunidade São Cristovão, CEP 85660-000 Dois Vizinhos, PR, Brasil. E-mail: domenico@utfpr.edu.br, moesesdanner@utfpr.edu.br, cleversonbusso@utfpr.edu.br (2)Universidade Estadual do Oeste do Paraná, Campus Cascavel, Rua Universitária, oo 2.069, CEP 85819-110 Cascavel, PR, Brasil. E-mail: divair.christ@unioeste.br, silvia.coelho@unioeste.br
\end{abstract}

Resumo - O objetivo deste trabalho foi determinar a magnitude dos efeitos diretos e indiretos de parâmetros físico-químicos e microbiológicos sobre a ocorrência de aflatoxinas em grãos de milho armazenados, por meio da análise de trilha. Os dados foram coletados em dois experimentos: um cultivo de inverno (colheita do milho em julho de 2012) e um cultivo de verão (colheita em fevereiro de 2013). Os grãos foram armazenados por 12 meses, em dois tipos de acondicionamentos: em sacarias convencionais de polipropileno e a granel em silo metálico. Avaliaram-se 14 características dos grãos. Calculou-se a matriz de correlações de Pearson entre todos os parâmetros, realizou-se o diagnóstico de multicolinearidade e, posteriormente, aplicou-se a análise de trilha com regressão em crista. Observou-se forte influência das condições ambientais entre as safras de inverno e de verão, e entre época e tipo de armazenagem, nos coeficientes de análise de trilha. As variáveis teor de umidade, conteúdo de cinzas, proteínas, lipídios, grãos sem defeitos, massa volumétrica, incidência de Penicillium sp., Aspergillus sp. e Fusarium sp. devem ser consideradas conjuntamente na definição da possibilidade de ocorrência de aflatoxinas na massa de grãos armazenados.

Termos para indexação: Zea mays, armazenagem, micotoxinas, multicolinearidade.

\section{Path analysis of aflatoxin contamination on stored maize kernels}

\begin{abstract}
The objective of this work was to determine the magnitude of the direct and indirect effects of the physicochemical and microbiological parameters on the occurrence of aflatoxins on stored maize kernels, through path analysis. Data were collected in two experiments: one winter crop (maize harvest in July 2012) and one summer crop (harvest in February 2013). Grains were stored for 12 months in two types of packaging: in conventional polypropylene sacks and bulk storage in metallic silo. Fourteen grain characteristics were evaluated. The matrix of Pearson's correlations was calculated between all parameters, the diagnosis of multicollinearity was performed, and subsequently path analysis was applied with ridge regression. A strong influence of environmental conditions was observed between winter and summer crops, and between season and type of storage, in path analysis coefficients. The variables moisture content, ash, proteins, lipids, undamaged kernels, volumetric weight, incidence of Penicillium sp., Aspergillus sp., and Fusarium sp. should be considered together for defining the probability of aflatoxin occurrence in the bulk of stored kernels.
\end{abstract}

Index terms: Zea mays, storage, mycotoxins, multicollinearity.

\section{Introdução}

O milho (Zea mays L.) é um dos principais cereais produzidos no mundo e o segundo grão mais produzido no Brasil, com produção acima de 80 milhões de toneladas na safra 2013/2014 (Companhia Nacional de Abastecimento, 2015). Em razão de sua riqueza nutricional, é utilizado na alimentação humana e animal, e é o ingrediente predominante na formulação de rações. Para atender às demandas ao longo do ano, este cereal fica armazenado e, portanto, predisposto à perda de qualidade e a contaminações fúngicas (Schuh et al., 2011). As implicações são: perda de massa, de volume, de vigor, da qualidade de moagem, degradação nutricional, descoloramento, odor desagradável, aquecimento, mudanças químicas e produção de micotoxinas (Faroni et al., 2005; Hermanns et al., 2006).

O armazenamento é uma das etapas pós-colheita mais longas e contribui decisivamente para a qualidade final 
dos grãos. A manutenção da qualidade ou a aceleração da degradação, durante esta etapa, é influenciada pela eficiência do processo de secagem, teor de umidade dos grãos, atmosfera e período de armazenagem, controle de pragas, temperatura e umidade relativa do ar, percentual de grãos danificados, presença de impurezas, matérias estranhas, microrganismos, insetos e ácaros (Magan \& Aldred, 2007).

Por ser rico em amido, o milho é um dos cereais mais vulneráveis ao desenvolvimento de fungos e à contaminação pormicotoxinas, metabólitos secundários tóxicos produzidos por fungos filamentosos, principalmente os dos gêneros Aspergillus, Penicillium e Fusarium (Marín et al., 1998a; Marques et al., 2009). A contaminação pode ocorrer no campo ou durante armazenagem ou, ainda, após o processamento (Ono et al., 2006). Muitos fatores podem contribuir para a infecção do milho por micotoxinas como: genótipo, condições ambientais, momento de colheita, danos mecânicos, tempo entre colheita, secagem e armazenagem e condições de armazenagem (Paterson \& Lima, 2010).

Entre as principais micotoxinas detectadas em milho estão as aflatoxinas $B_{1}, B_{2}, G_{1}$ e $G_{2}$, produzidas por diversas espécies do gênero Aspergillus. Elas geram efeitos tóxicos em humanos, pois têm potencial carcinogênico, mutagênico, imunossupressor e teratogênico (Maziero \& Bersot, 2010). Podem interferir na exportação de grãos ou impedi-la e reduzir a produtividade pecuária por causar intoxicação e até morte de animais (Mulunda et al., 2013).

Em razão dos problemas causados pelas micotoxinas à cadeia alimentar, alguns trabalhos foram realizados para estudar as correlações entre estas e as variáveis ambientais, físico-químicas e da incidência de fungos em grãos de milho recém-colhidos ou armazenados (Ono et al., 2006; Almeida et al., 2009; Bento et al., 2012; Tédihou et al., 2012). As correlações simples quantificam o grau e o sentido da associação linear existente entre duas variáveis, porém, não garantem uma relação de causa e efeito entre o grupo de variáveis envolvidas em relação a uma variável dependente de maior importância (Lúcio et al., 2013). Uma das desvantagens é que um alto ou baixo coeficiente de correlação pode resultar do efeito de outras variáveis (Oliveira et al., 2010). No entanto, a análise de trilha - desenvolvida por Wright (1921) - possibilita o desdobramento do coeficiente de correlação em efeitos diretos e indiretos de um grupo de variáveis (variáveis explicativas) sobre uma variável principal (básica). Isto resulta no estudo da relação de causa e efeito entre as variáveis. Os coeficientes de trilha são obtidos por meio de equações de regressão em que as variáveis são previamente padronizadas (Rios et al., 2012).

Antes da aplicação desta análise, deve-se fazer o diagnóstico de multicolinearidade, pois problemas com esta podem fazer com que os resultados não sejam confiáveis. Caso se verifique a presença de multicolinearidade, Cruz \& Carneiro (2006) recomendam duas alternativas: a primeira é identificar e excluir as variáveis que estão provocando a colinearidade, e a segunda, caso o pesquisador não queira eliminar variáveis, deve adotar a metodologia denominada análise de regressão em crista. A análise de trilha pode ser aplicada para gerar mais informações, por causa da escassez de informações em relação aos parâmetros que merecem maior atenção na produção de aflatoxinas em grãos de milho armazenados.

O objetivo deste trabalho foi determinar a magnitude dos efeitos diretos e indiretos de parâmetros físico-químicos e microbiológicos sobre a variável ocorrência de aflatoxinas de grãos de milho armazenados, por meio da análise de trilha.

\section{Material e métodos}

Foram utilizados dados obtidos de dois experimentos anuais de armazenagem de grãos de milho, realizados na Universidade Tecnológica Federal do Paraná

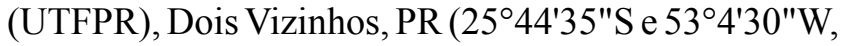
a $509 \mathrm{~m}$ de altitude). Os grãos utilizados são do híbrido de milho 2B688RR, em ambos os experimentos. No experimento I, os grãos foram colhidos na safra de inverno (28/6/2012) e armazenados de julho/2012 a julho/2013 (365 dias). No experimento II, os grãos foram colhidos na safra de verão (18/2/2013) e armazenados de fevereiro/2013 a fevereiro/2014 (365 dias).

Após a colheita mecânica dos grãos, realizouse secagem natural até que o teor de umidade fosse menor ou igual a $13 \%$, seguida de limpeza em máquina classificadora e armazenagem, por 12 meses, em uma sala de alvenaria em condições ambientais. Utilizaramse duas formas de armazenagem: em sacaria de polipropileno $(38 \times 52 \mathrm{~cm})$, com $4 \mathrm{~kg}$ de grãos cada, empilhadas sobre estrados de madeira (30 unidades 
divididas em 6 pilhas); e armazenamento a granel, em silos metálicos ( 6 unidades de $0,35 \mathrm{~m}$ de diâmetro por $0,6 \mathrm{~m}$ de altura), sem sistema de aeração, com $25 \mathrm{~kg}$ de grãos cada.

Ambos os experimentos foram conduzidos em delineamento inteiramente casualizado, com três repetições, em arranjo fatorial $2 \times 5$, cujos fatores foram formas (sacaria e silo metálico) e períodos de armazenamento (0, 3, 6, 9 e 12 meses). Em cada período, realizou-se coleta de três amostras, por forma de armazenagem, tendo-se retirado aleatoriamente três sacos da pilha ou coletando-se grãos por meio de um calador graneleiro nos silos metálicos. As análises foram realizadas em triplicata e avaliaram-se 14 atributos: teor de umidade, conteúdo de cinzas, proteínas e lipídios, grãos sem defeitos, massa volumétrica e massa de mil grãos, incidência de Penicillium sp., Aspergillus sp. e Fusarium sp., contagem total de bolores e leveduras, ocorrência de aflatoxinas (dos tipos $B_{1}, B_{2}, G_{1}$ e $G_{2}$ ) e média diária de temperatura e de umidade relativa do ar.

$\mathrm{O}$ teor de umidade (\%) foi determinado pelo método-padrão da estufa a $105 \pm 3^{\circ} \mathrm{C}$, durante 24 horas (Regras..., 2009). O conteúdo de cinzas (\%) foi determinado por incineração em mufla a $550{ }^{\circ} \mathrm{C}$, o teor proteínas (\%) foi medido pelo método Kjedahl, e os lipídios (\%) por extração em Soxhlet, de acordo com as técnicas 018, 036, 032/IV, respectivamente (Zenebon et al., 2008). A percentagem de grãos sem defeitos foi calculada subtraindo-se de 100 a percentagem de grãos carunchados, avariados, ardidos, quebrados, fermentados, germinados, mofados, chochos e/ou imaturos, com impurezas ou matérias estranhas (Brasil, 1976). A massa volumétrica e a massa de mil grãos foram determinadas segundo as Regras para Análise de Sementes (Regras..., 2009).

Para a análise microbiológica, foram moídas $200 \mathrm{~g}$ de cada amostra de milho e passadas em peneira 50 mesh. Uma subamostra de $10 \mathrm{~g}$ foi misturada com $90 \mathrm{~mL}$ de água peptonada estéril a $0,1 \%$. Em seguida, foram realizadas diluições em série até $10^{-5}$. Um mililitro de cada diluição foi transferido para placas de Petri por meio da técnica "Pour Plate" com ágar batata dextrose (BDA, $\mathrm{pH} 4,0$, acidificado com ácido tartárico a $10 \%$ ), que foram incubadas a $25^{\circ} \mathrm{C}$, por 5 a 7 dias. Após a contagem total de fungos (UFC $\mathrm{g}^{-1}$ ), realizouse a identificação dos gêneros de acordo com Singh et al. (1991), com base nos aspectos macroscópicos das colônias e microscópicos das estruturas vegetativas e reprodutoras.

A análise de aflatoxinas $B_{1}, B_{2}, G_{1}$ e $G_{2}$ foi realizada por cromatografia líquida de alta eficiência (Clae) segundo a metodologia descrita pelo fabricante das colunas de imunoafinidade (Aflatest VICAM). A extração foi efetuada com $50 \mathrm{~g}$ da amostra moída, e o solvente utilizado foi metanol-água $(80-20, \mathrm{v} / \mathrm{v})$. A purificação ocorreu pela passagem do extrato pelas colunas de imunoafinidade, e a derivatização foi feita com ácido trifluoracético:ácido acético:água (2:1:7). Para a análise, injetaram-se $20 \mu \mathrm{L}$ do eluato no sistema Clae (Shimadzu Corp., Kyoto, Japan), composto de injetor automático, bomba quaternária, forno de colunas ajustado a $40^{\circ} \mathrm{C}$, coluna cromatográfica $\mathrm{C} 18$ $(5 \mu \mathrm{m}) 150 \times 4,6 \mathrm{~mm}$ e detector de fluorescência, operado em $365 \mathrm{~nm}$ de excitação e $450 \mathrm{~nm}$ de emissão. A fase móvel utilizada foi a mistura isocrática de água-metanol $(60-40, \mathrm{v} / \mathrm{v})$ ao fluxo de $0,8 \mathrm{~mL}$ por minuto. As identificações e quantificações $\left(\mu \mathrm{g} \mathrm{kg}^{-1}\right)$ foram realizadas por meio de curvas de calibração, obtidas com os padrões $\mathrm{B}_{1}, \mathrm{~B}_{2}, \mathrm{G}_{1} \mathrm{e}_{2}$ (Sigma-Aldrich) diluídos em metanol grau HPLC, por meio de regressão linear. O tempo de corrida cromatográfica por amostra foi de $15 \mathrm{~min}$, com três leituras para cada corrida, e os tempos de retenção foram 6,2, 7,9, 10,8 e 12,3 min para $G_{2}, G_{1}, B_{2}$ e $B_{1}$, respectivamente.

Durante os experimentos de armazenagem (julho/2012 a julho/2013 e fevereiro/2013 a fevereiro/2014), as condições diárias de temperatura e umidade relativa do ar foram registradas pela estação meteorológica do Instituto Nacional de Meteorologia, instalada no campus da universidade, cujas médias mensais estão apresentadas na Figura 1. Não se realizou tratamento prévio com inseticida nos grãos, mas, durante o experimento, foi necessário o uso de medidas curativas, como o uso de fumigantes (Gastoxin B57) para o controle de insetos, e armadilhas e raticidas (Citromax) para o controle de roedores.

Para a análise dos dados, foi considerada cada uma das análises no tempo de armazenagem, separadas por experimento (I e II) e por forma de armazenamento (sacaria e silo metálico). Estimaramse os coeficientes de correlação de Pearson (r), cuja significância foi determinada pelo teste $t$ de Student, a 5\% de probabilidade. Aplicou-se o teste de Lilliefors, para verificar a normalidade dos dados de cada parâmetro e, como estes se ajustaram, não foi 
necessária a transformação. Em seguida, procedeuse ao diagnóstico de multicolinearidade, pela análise do número de condição (NC), que representa a razão entre o maior e o menor autovalor da matriz de correlações. Segundo Montgomery \& Peck (1981), se $\mathrm{NC}<100$, a colinearidade é fraca, se $100<\mathrm{NC}<1.000$ é de moderada a forte, e se $\mathrm{NC}>1.000$, é severa. Depois de constatar multicolinearidade severa em todos os diagnósticos, excluíram-se quatro variáveis (massa de mil grãos, contagem total de bolores e leveduras, temperatura e umidade relativa do ar). Mesmo após a exclusão, a multicolinearidade estava de moderada a forte. Por isso, realizou-se a análise de regressão em crista ou análise de trilha sob multicolinearidade com as 10 variáveis restantes. Neste procedimento, utilizase uma constante $\mathrm{k}$ (cujo valor deve ser o menor possível), para estabilizar os coeficientes de trilha, mantendo-se o fator de inflação da variância (VIF) menor que 10, em todas as variáveis explicativas (Cruz \& Carneiro, 2006; Rios et al., 2012). A eliminação de mais variáveis foi evitada, pois propiciaria redução do coeficiente de determinação $\left(\mathrm{R}^{2}\right)$. Todas as análises foram realizadas com o programa Genes (Cruz, 2006).

A interpretação dos efeitos dos coeficientes de trilha foi relacionada ao coeficiente de correlação de

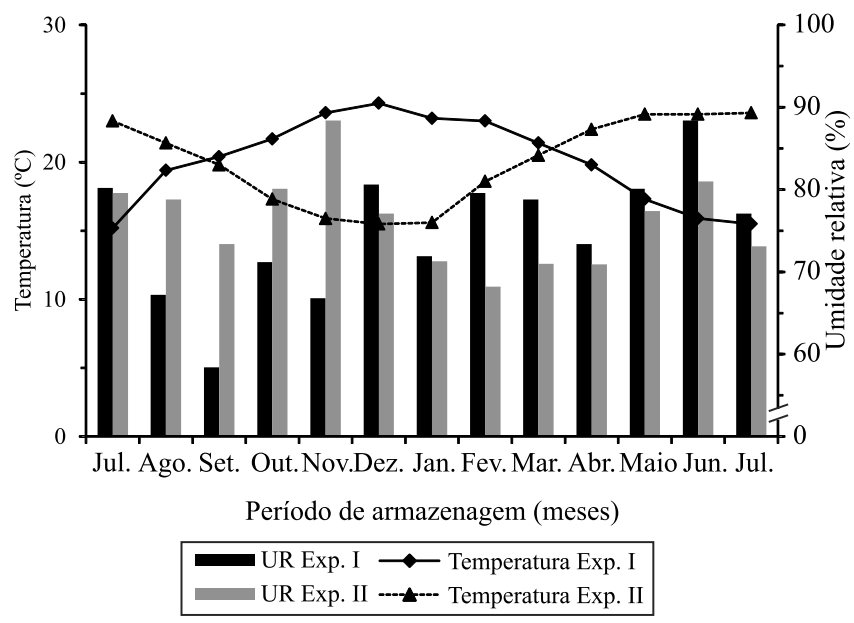

Figura 1. Temperatura média mensal e umidade relativa do ar média mensal durante os 12 meses de armazenamento, para os dois experimentos. Experimento I (Exp. I): realizado de julho/2012 a julho/2013; experimento II (Exp. II), realizado de fevereiro/2013 a fevereiro/2014.
Pearson, conforme Lúcio et al. (2013): quando r e o efeito direto são iguais ou semelhantes, em magnitude e sinal, a correlação explica bem a associação entre as variáveis; se r é positivo, e o efeito direto desprezível ou negativo, a correlação é ocasionada pelos efeitos indiretos; se $r$ for desprezível, e o efeito direto positivo e alto, são os efeitos indiretos que causam a falta de correlação; se r é negativo, e o efeito direto é positivo e alto, os efeitos indiretos devem ser e somente os diretos devem ser considerados.

\section{Resultados e Discussão}

Em sua maioria, os parâmetros avaliados, apresentaram coeficientes de correlação linear de Pearson significativos e de magnitude média $(0,385 \leq|\mathrm{r}|<0,6)$, o que implica grau médio de interrelações entre as variáveis (Carvalho et al., 2004). As maiores correlações significativas foram encontradas entre as variáveis massa volumétrica e grãos sem defeitos, que foram de 0,72 e 0,90 no experimento I e de 0,75 e 0,89 no experimento II, na armazenagem com sacarias e em silos metálicos, respectivamente (Tabela 1). O resultado apresentado já era esperado, pois, quanto maior a percentagem de grãos sem defeitos, maior o número de grãos sadios (inteiros) e maior será a massa volumétrica. A variável base - ocorrência de aflatoxinas nos grãos de milho - do experimento I apresentou correlações significativas positivas apenas com o conteúdo de lipídios $(0,52)$ e com a incidência de Aspergillus sp. $(0,59)$, na armazenagem em sacaria convencional, e com o conteúdo de proteínas $(0,43)$ na armazenagem em silos metálicos. No experimento II, a ocorrência de aflatoxinas foi correlacionada significativamente à maioria das variáveis, principalmente na armazenagem com silo metálico (Tabela 1). A falta de correlação das aflatoxinas com as demais variáveis, no experimento I, pode estar relacionada à baixa incidência fúngica, principalmente do gênero Aspergillus.

$\mathrm{Na}$ análise de trilha, verificou-se falta de correlação do teor de umidade dos grãos com a ocorrência de aflatoxinas em ambos os experimentos, com ambas as formas de armazenamento, em razão dos vários efeitos indiretos, principalmente pela incidência dos fungos dos três gêneros (Tabela 2). Almeida et al. (2009) também não obtiveram correlação significativa entre o teor de umidade dos grãos de milho (que foi $<14 \%$ ) e a 
presença de aflatoxinas. Segundo Leeson et al. (1995), para o crescimento de fungos toxigênicos do gênero Aspergillus e a produção de aflatoxinas, a condição ideal é de que o teor de umidade dos grãos de milho seja de $17 \%$. No presente estudo, o grau de umidade dos grãos de milho variou de 11,12 a 14,61\%.

O conteúdo de cinzas apresentou alto efeito direto sobre a ocorrência de aflatoxinas, em ambos os tipos de armazenamento do experimento I e na sacaria do experimento II, mas o coeficiente de correlação de Pearson foi baixo e não significativo (Tabela 2). Isto também indica que são os efeitos indiretos que causam a falta de correlação. Schuh et al. (2011) verificaram que o consumo de componentes orgânicos, pela atividade metabólica dos grãos e dos fungos associados, causou aumento do teor de cinzas durante o armazenamento. Porém, a relação de aumento do conteúdo de cinzas dos grãos de milho com o aumento da ocorrência de aflatoxinas não é suportada pelos resultados do presente trabalho.

Os resultados da análise de trilha quanto ao conteúdo de proteínas também revelaram que os efeitos indiretos causam a falta de correlação com a ocorrência de aflatoxina. O conteúdo de lipídios dos grãos de milho apresentou correlação linear $(\mathrm{r}=0,5163)$ e efeito direto $(0,6887)$, com mesmo sinal e magnitude semelhantes nas sacarias convencionais do experimento I (safra de inverno), o que evidencia uma relação de causa e efeito entre esta variável e a ocorrência de aflatoxinas. Entretanto, no armazenamento em silo metálico, do experimento I, e em ambos os tipos de armazenamento do experimento II, este efeito não foi verificado, o que mostra que há interferência do tipo de armazenamento e das safras sobre as correlações e magnitudes dos efeitos. Almeida et al. (2009) não encontraram correlação significativa entre a presença de aflatoxinas e o conteúdo de lipídios. Entretanto, Saleemullah et al. (2006) constataram grande redução do conteúdo de proteínas, lipídios, cinzas e carboidratos, em amostras de pipoca e grãos de milho infectados com aflatoxinas durante o armazenamento. Esta disparidade entre os resultados de Almeida et al. (2009) e de Saleemullah et al. (2006), em relação ao conteúdo de aflatoxinas e de lipídios dos grãos, é corroborada pelos diferentes

Tabela 1. Estimativas das correlações de Pearson (r) entre as variáveis analisadas em grãos de milho dos experimentos I, na safra de inverno com armazenamento de julho de 2012 a julho de 2013 (dados acima da diagonal), e II, na safra de verão com armazenamento de fevereiro de 2013 a fevereiro 2014 (dados abaixo da diagonal), nos armazenamentos em sacaria convencional (ASC) e em silos metálicos (ASM).

\begin{tabular}{|c|c|c|c|c|c|c|c|c|c|c|c|}
\hline \multicolumn{2}{|c|}{ Variáveis $^{(1)}$} & \multirow{2}{*}{$\begin{array}{c}\mathrm{TU} \\
-\end{array}$} & \multirow{2}{*}{$\begin{array}{c}\mathrm{CC} \\
0,459^{*}\end{array}$} & \multirow{2}{*}{\begin{tabular}{|c|}
$\mathrm{CP}$ \\
$-0,172$
\end{tabular}} & \multirow{2}{*}{$\begin{array}{c}\mathrm{CL} \\
-0,514^{* *}\end{array}$} & \multirow{2}{*}{$\begin{array}{c}\text { GSD } \\
-0,719^{* *}\end{array}$} & \multirow{2}{*}{$\begin{array}{c}\text { PV } \\
-0,567^{*}\end{array}$} & \multirow{2}{*}{$\begin{array}{c}\mathrm{IP} \\
-0,122\end{array}$} & \multirow{2}{*}{\begin{tabular}{|c|} 
IA \\
0,241
\end{tabular}} & \multirow{2}{*}{$\begin{array}{c}\text { IF } \\
-0,477^{*}\end{array}$} & \multirow{2}{*}{\begin{tabular}{|c|}
$\mathrm{AFL}$ \\
$-0,178$
\end{tabular}} \\
\hline & ASC & & & & & & & & & & \\
\hline$T U$ & ASM & - & $-0,337$ & $-0,69 * *$ & $-0,288$ & $-0,389 *$ & $-0,348$ & 0,205 & 0,284 & $0,451^{*}$ & $-0,112$ \\
\hline \multirow{2}{*}{$\mathrm{CC}$} & ASC & $-0,006$ & - & $0,454 *$ & 0,110 & $-0,587 * *$ & $-0,429 *$ & 0,208 & 0,196 & 0,046 & 0,152 \\
\hline & ASM & $0,869 * *$ & - & $0,553 * *$ & 0,088 & $-0,580 * *$ & $-0,651^{* *}$ & $-0,337$ & 0,034 & $-0,261$ & 0,270 \\
\hline \multirow{2}{*}{$\mathrm{CP}$} & ASC & $-0,069$ & 0,130 & - & $0,525^{* *}$ & $-0,064$ & $-0,420^{*}$ & 0,138 & 0,040 & 0,129 & 0,327 \\
\hline & ASM & $0,796 * *$ & $0,686 * *$ & - & 0,157 & 0,128 & $-0,0003$ & $-0,377$ & 0,109 & $-0,232$ & $0,434 *$ \\
\hline \multirow{2}{*}{$\mathrm{CL}$} & $\mathrm{ASC}$ & 0,092 & $0,779 * *$ & 0,219 & - & 0,115 & $-0,196$ & 0,032 & $-0,033$ & 0,484* & $0,516^{* *}$ \\
\hline & ASM & $0,424^{*}$ & $0,661 * *$ & 0,345 & - & 0,198 & 0,022 & $0,406^{*}$ & 0,002 & 0,083 & $-0,080$ \\
\hline \multirow{2}{*}{ GSD } & ASC & 0,063 & $-0,558 * *$ & $-0,100$ & $-0,707 * *$ & - & $0,722 * *$ & 0,140 & $-0,335$ & 0,362 & 0,004 \\
\hline & ASM & $-0,709 * *$ & $-0,565 * *$ & $-0,388^{*}$ & $-0,079$ & - & $0,905^{* *}$ & $-0,014$ & 0,013 & $-0,230$ & 0,167 \\
\hline \multirow{2}{*}{ PV } & ASC & $-0,206$ & $-0,693 * *$ & 0,140 & $-0,540 * *$ & $0,755^{* *}$ & - & 0,204 & $-0,478^{*}$ & 0,255 & $-0,252$ \\
\hline & ASM & $-0,786^{* *}$ & $-0,662 * *$ & $-0,543 * *$ & $-0,069$ & $0,891^{* *}$ & - & 0,027 & 0,045 & $-0,207$ & 0,094 \\
\hline \multirow{2}{*}{ IP } & ASC & $-0,558 * *$ & 0,089 & $-0,213$ & $-0,256$ & 0,358 & 0,240 & - & $-0,136$ & 0,300 & 0,184 \\
\hline & ASM & $0,400 *$ & 0,257 & 0,367 & $-0,128$ & $-0,364$ & $-0,449 *$ & - & $-0,161$ & 0,357 & $-0,204$ \\
\hline \multirow{2}{*}{ IA } & $\mathrm{ASC}$ & 0,185 & 0,215 & $-0,344$ & $-0,332$ & $0,449^{*}$ & $-0,167$ & $0,488^{*}$ & - & $-0,130$ & 0,192 \\
\hline & ASM & $0,422 *$ & 0,313 & 0,256 & $-0,017$ & $-0,461 *$ & $-0,548 * *$ & $0,887 * *$ & - & $-0,189$ & $0,594 * *$ \\
\hline \multirow{2}{*}{ IF } & ASC & $-0,106$ & $-0,187$ & $-0,187$ & $-0,647 * *$ & $0,782^{* *}$ & $0,394^{*}$ & $0,530^{* *}$ & $0,705^{* *}$ & - & $-0,097$ \\
\hline & ASM & $-0,632 * *$ & $-0,402 *$ & $-0,476^{*}$ & $-0,303$ & $0,565^{* *}$ & $0,595 * *$ & $-0,148$ & $-0,239$ & - & $-0,318$ \\
\hline \multirow{2}{*}{ AFL } & ASC & $-0,307$ & 0,009 & 0,050 & $-0,145$ & $0,462^{*}$ & $0,530^{* *}$ & $0,657 * *$ & 0,124 & $0,523 * *$ & - \\
\hline & ASM & $-0,531 * *$ & $-0,446^{*}$ & $-0,317$ & $-0,394 *$ & $0,485^{*}$ & $0,487 *$ & $-0,366$ & $-0,656^{* *}$ & $0,608 * *$ & - \\
\hline
\end{tabular}

${ }^{(1)} \mathrm{TU}$, teor de umidade (\%); CC, conteúdo de cinzas (\%); CP, conteúdo de proteínas (\%); CL, conteúdo de lípidios (\%); GSD, grãos sem defeitos (\%); PV, massa volumétrica $\left(\mathrm{kg} \mathrm{m}^{-3}\right)$; IP, incidência de Penicillium sp. ( $\left.\mathrm{x}^{-4} \mathrm{UFC} \mathrm{g}^{-1}\right)$; IA, incidência de Aspergillus sp. ( $\left.\times 10^{-4} \mathrm{UFC} \mathrm{g}^{-1}\right)$; IF, incidência de Fusarium sp. $\left(\mathrm{x} 10^{-4} \mathrm{UFC} \mathrm{g}^{-1}\right)$; AFL, soma de aflatoxinas $\mathrm{B}_{1,} \mathrm{~B}_{2}, \mathrm{G}_{1} \mathrm{e} \mathrm{G}_{2} \mathrm{em} \mu \mathrm{g} \mathrm{kg}{ }^{-1}$. ** e *Significativo a $1 \mathrm{e} 5 \%$ de probabilidade, pelo teste $\mathrm{t}$. 
Tabela 2. Efeitos diretos (ED) e indiretos (EI), e coeficientes de correlações de Pearson (r) dos parâmetros (variáveis explicativas) teor de umidade (TU), conteúdo de cinzas (CC), conteúdo de proteínas $(\mathrm{CP})$, conteúdo de lipídios $(\mathrm{CL})$, grãos sem defeitos (GSD), massa volumétrica (PV), incidência de Penicillium sp., (IP), incidência de Aspergillus sp., (IA), e incidência de Fusarium sp., (IF), com a ocorrência de aflatoxinas $\mathrm{B}_{1}, \mathrm{~B}_{2}, \mathrm{G}_{1}$ e $\mathrm{G}_{2}(\mathrm{AFL})$. Armazenamentos em silos metálicos (ASM) e em sacaria convencional (ASC), nos experimentos I e II.

\begin{tabular}{|c|c|c|c|c|}
\hline \multirow[t]{3}{*}{ Efeito } & \multicolumn{2}{|c|}{ Experimento I } & \multicolumn{2}{|c|}{ Experimento II } \\
\hline & $\operatorname{ASM}(n=39)$ & $\operatorname{ASC}(n=39)$ & $\operatorname{ASM}(n=39)$ & $\operatorname{ASC}(n=39)$ \\
\hline & Estimativa $^{(1)}$ & Estimativa & Estimativa & Estimativa \\
\hline & \multicolumn{4}{|c|}{ Teor de umidade (TU) } \\
\hline ED sobre AFL & 0,2390 & $-0,3292$ & $-0,0583$ & 0,3750 \\
\hline $\mathrm{EI}$ via $\mathrm{CC}$ & $-0,2700$ & 0,2098 & 0,0009 & $-0,0029$ \\
\hline EI via $\mathrm{CP}$ & $-0,1248$ & 0,0754 & $-0,0623$ & 0,0016 \\
\hline EI via CL & 0,1150 & $-0,3539$ & $-0,0715$ & $-0,0008$ \\
\hline EI via GSD & $-0,3203$ & $-0,3863$ & $-0,1223$ & 0,0052 \\
\hline EI via PV & 0,2278 & 0,2913 & 0,2650 & $-0,0638$ \\
\hline EI via IP & 0,0682 & $-0,0414$ & 0,2554 & $-0,4227$ \\
\hline EI via IA & 0,1471 & 0,0267 & $-0,4876$ & $-0,1495$ \\
\hline EI via IF & 0,0057 & 0,3322 & $-0,2483$ & $-0,0637$ \\
\hline \multirow[t]{2}{*}{$\underline{\mathrm{R}}$} & $-0,112^{\mathrm{ns}}$ & $-0,178^{\mathrm{ns}}$ & $-0,531^{*}$ & $-0,307^{\mathrm{ns}}$ \\
\hline & \multicolumn{4}{|c|}{ Conteúdo de cinzas (CC) } \\
\hline ED sobre AFL & 0,8003 & 0,4566 & 0,0010 & 0,4821 \\
\hline EI via TU & $-0,0816$ & $-0,1512$ & $-0,0507$ & $-0,0022$ \\
\hline EI via $\mathrm{CP}$ & 0,1002 & $-0,1990$ & $-0,0537$ & $-0,0030$ \\
\hline EI via $C L$ & $-0,0352$ & 0,0761 & $-0,1114$ & $-0,0070$ \\
\hline EI via GSD & $-0,4773$ & $-0,3155$ & $-0,0975$ & $-0,0462$ \\
\hline EI via PV & 0,0426 & 0,2207 & 0,2228 & $-0,2148$ \\
\hline EI via IP & $-0,1121$ & 0,0705 & 0,1640 & 0,0674 \\
\hline EI via IA & 0,0173 & 0,0217 & $-0,3629$ & $-0,1737$ \\
\hline EI via IF & $-0,0033$ & $-0,0318$ & $-0,1579$ & $-0,1124$ \\
\hline \multirow[t]{2}{*}{$\underline{\mathrm{R}}$} & $0,2696^{\mathrm{ns}}$ & $0,152^{\mathrm{ns}}$ & $-0,446^{*}$ & $0,009^{\text {ns }}$ \\
\hline & \multicolumn{4}{|c|}{ Conteúdo de proteínas $(\mathrm{CP})$} \\
\hline ED sobre AFL & 0,1810 & $-0,4381$ & $-0,0782$ & $-0,0231$ \\
\hline EI via TU & $-0,1648$ & 0,0567 & $-0,0464$ & $-0,0259$ \\
\hline $\mathrm{EI}$ via $\mathrm{CC}$ & 0,4430 & 0,2074 & 0,00069 & 0,0627 \\
\hline EI via $C L$ & $-0,0626$ & 0,3618 & $-0,0582$ & $-0,0020$ \\
\hline EI via GSD & 0,1056 & $-0,0346$ & $-0,0669$ & $-0,0083$ \\
\hline EI via PV & 0,0000 & 0,2160 & 0,1831 & 0,0433 \\
\hline EI via IP & $-0,1254$ & 0,0469 & 0,2343 & $-0,1614$ \\
\hline EI via IA & 0,05638 & 0,0044 & $-0,2958$ & 0,2779 \\
\hline EI via IF & $-0,0029$ & $-0,0896$ & $-0,1870$ & $-0,1124$ \\
\hline \multirow[t]{2}{*}{$\underline{\mathrm{R}}$} & $0,4341 *$ & $0,3271^{\mathrm{ns}}$ & $-0,317^{\mathrm{ns}}$ & $0,05^{\mathrm{ns}}$ \\
\hline & \multicolumn{4}{|c|}{ Conteúdo de lipídios (CL) } \\
\hline ED sobre AFL & $-0,3997$ & 0,6887 & $-0,1686$ & $-0,009$ \\
\hline EI via TU & $-0,0687$ & 0,1692 & $-0,0247$ & 0,0344 \\
\hline $\mathrm{EI}$ via $\mathrm{CC}$ & 0,0705 & 0,0545 & 0,0007 & 0,3756 \\
\hline EI via $\mathrm{CP}$ & 0,0284 & $-0,2302$ & $-0,0270$ & $-0,0050$ \\
\hline EI via GSD & 0,1632 & 0,0616 & $-0,0136$ & $-0,0586$ \\
\hline EI via PV & $-0,0014$ & 0,1006 & 0,0232 & $-0,1673$ \\
\hline EI via IP & 0,1347 & 0,0108 & $-0,0817$ & $-0,1939$ \\
\hline EI via IA & 0,0011 & $-0,0037$ & 0,0208 & 0,2682 \\
\hline EI via IF & 0,0010 & $-0,3372$ & $-0,1190$ & $-0,3889$ \\
\hline \multirow[t]{2}{*}{$\underline{\mathrm{R}}$} & $-0,0798^{\mathrm{ns}}$ & $0,5163 * *$ & $-0,395^{*}$ & $-0,145^{\mathrm{ns}}$ \\
\hline & \multicolumn{4}{|c|}{ Grãos sem defeitos (GSD) } \\
\hline ED sobre AFL & 0,8227 & 0,5373 & 0,1725 & 0,0828 \\
\hline EI via TU & $-0,0930$ & 0,2367 & 0,0414 & 0,0236 \\
\hline
\end{tabular}

Continua........
Continuação.....

\begin{tabular}{|c|c|c|c|c|}
\hline EI via $\mathrm{CC}$ & $-0,4643$ & $-0,2681$ & $-0,0006$ & $-0,2691$ \\
\hline EI via $\mathrm{CP}$ & 0,0232 & 0,0282 & 0,0303 & 0,0023 \\
\hline EI via $\mathrm{CL}$ & $-0,0793$ & 0,0789 & 0,0133 & 0,0064 \\
\hline EI via PV & $-0,0593$ & $-0,3714$ & $-0,3000$ & 0,2339 \\
\hline EI via IP & $-0,0047$ & 0,0473 & $-0,2324$ & 0,2712 \\
\hline EI via IA & 0,0065 & $-0,0371$ & 0,5327 & $-0,3627$ \\
\hline EI via IF & $-0,0029$ & $-0,2521$ & 0,2220 & 0,4700 \\
\hline \multirow[t]{2}{*}{$\mathrm{R}$} & $0,167^{\mathrm{ns}}$ & $0,004^{\mathrm{ns}}$ & $0,485^{*}$ & $0,462 *$ \\
\hline & \multicolumn{4}{|c|}{ Massa volumétrica (PV) } \\
\hline ED sobre AFL & $-0,0655$ & $-0,5141$ & $-0,3367$ & 0,3099 \\
\hline EI via TU & $-0,0831$ & 0,1865 & 0,0459 & $-0,0772$ \\
\hline EI via CC & $-0,5212$ & $-0,1961$ & $-0,0007$ & $-0,3341$ \\
\hline EI via $\mathrm{CP}$ & $-0,0000$ & 0,1841 & 0,0425 & $-0,0032$ \\
\hline EI via $C L$ & $-0,0088$ & $-0,1347$ & 0,0116 & 0,0048 \\
\hline EI via GSD & 0,7446 & 0,3881 & 0,1537 & 0,0625 \\
\hline EI via IP & 0,0089 & 0,0692 & $-0,2866$ & 0,1826 \\
\hline EI via IA & 0,0235 & $-0,0529$ & 0,6333 & 0,1357 \\
\hline EI via IF & $-0,0026$ & $-0,1777$ & 0,2342 & 0,2368 \\
\hline \multirow[t]{2}{*}{$\mathrm{R}$} & $0,094^{\text {ns }}$ & $-0,252^{\mathrm{ns}}$ & $0,487 *$ & $0,53 * *$ \\
\hline & \multicolumn{4}{|c|}{ Incidência de Penicillium sp. (IP) } \\
\hline ED sobre AFL & 0,3321 & 0,3389 & 0,6383 & 0,7576 \\
\hline EI via TU & 0,0491 & 0,0402 & $-0,0233$ & $-0,2092$ \\
\hline EI via $\mathrm{CC}$ & $-0,2701$ & 0,0950 & 0,0002 & 0,0429 \\
\hline EI via $\mathrm{CP}$ & $-0,0683$ & $-0,0606$ & $-0,0287$ & 0,0049 \\
\hline EI via $\mathrm{CL}$ & $-0,1621$ & 0,0219 & 0,0215 & 0,0023 \\
\hline EI via GSD & $-0,0116$ & 0,0751 & $-0,0628$ & 0,0296 \\
\hline EI via PV & $-0,0017$ & $-0,1050$ & 0,1511 & 0,0747 \\
\hline EI via IA & $-0,0832$ & $-0,0151$ & $-1,0250$ & $-0,3943$ \\
\hline EI via IF & 0,0045 & $-0,2091$ & $-0,0585$ & 0,3186 \\
\hline \multirow[t]{2}{*}{$\mathrm{R}$} & $-0,204^{\mathrm{ns}}$ & $0,184^{\text {ns }}$ & $-0,367^{\mathrm{ns}}$ & $0,657 * *$ \\
\hline & \multicolumn{4}{|c|}{ Incidência de Aspergillus sp. (IA) } \\
\hline ED sobre AFL & 0,5173 & 0,1107 & $-1,1556$ & $-0,8080$ \\
\hline EI via TU & 0,0680 & $-0,0794$ & $-0,0246$ & 0,0694 \\
\hline EI via $\mathrm{CC}$ & 0,0268 & 0,0894 & 0,0003 & 0,1037 \\
\hline EI via $\mathrm{CP}$ & 0,0197 & $-0,0175$ & $-0,0200$ & 0,0079 \\
\hline EI via $C L$ & $-0,0009$ & $-0,0228$ & 0,0030 & 0,0030 \\
\hline EI via GSD & 0,0103 & $-0,1799$ & $-0,0795$ & 0,0372 \\
\hline EI via PV & $-0,0030$ & 0,2456 & 0,1845 & $-0,0521$ \\
\hline EI via IP & $-0,0534$ & $-0,0462$ & 0,5662 & 0,3697 \\
\hline EI via IF & $-0,0024$ & 0,0908 & $-0,0939$ & 0,4244 \\
\hline \multirow[t]{2}{*}{$\mathrm{R}$} & $0,5937^{* *}$ & $0,1921^{\mathrm{ns}}$ & $-0,656 * *$ & $0,124^{\text {ns }}$ \\
\hline & \multicolumn{4}{|c|}{ Incidência de Fusarium sp. (IF) } \\
\hline ED sobre AFL & 0,0126 & $-0,6965$ & 0,3929 & 0,6011 \\
\hline EI via TU & 0,1079 & 0,1570 & 0,0368 & $-0,0397$ \\
\hline EI via $\mathrm{CC}$ & $-0,2090$ & 0,0209 & $-0,0004$ & $-0,0902$ \\
\hline EI via $\mathrm{CP}$ & $-0,0421$ & $-0,0563$ & 0,0372 & 0,0043 \\
\hline EI via $C L$ & $-0,0333$ & 0,3333 & 0,0511 & 0,0058 \\
\hline EI via GSD & $-0,1891$ & 0,1945 & 0,0974 & 0,0648 \\
\hline EI via PV & 0,0135 & $-0,1311$ & $-0,2007$ & 0,1221 \\
\hline EI via IP & 0,1186 & 0,1018 & $-0,0951$ & 0,4015 \\
\hline EI via IA & $-0,0976$ & $-0,0144$ & 0,2762 & $-0,5704$ \\
\hline$\underline{R}$ & $-0,3183^{\mathrm{ns}}$ & $-0,0969^{\text {ns }}$ & $0,608 *$ & $0,523 * *$ \\
\hline$\overline{\mathrm{NC}}$ & 105,12 & 105,03 & 222,33 & 379,45 \\
\hline $\mathrm{K}$ & 0,0220 & 0,0872 & 0,0316 & 0,0392 \\
\hline $\mathrm{R}^{2}$ & 0,6661 & 0,6235 & 0,8043 & 0,8039 \\
\hline Efeito residual & 0,5778 & 0,6135 & 0,4423 & 0,4428 \\
\hline
\end{tabular}

${ }^{(1)}$ Os fatores de inflação da variância (VIF) foram todos menores que 9,98 , na análise de trilha com multicolinearidade. ${ }^{\text {ns Não significativo. } * *}$ e *Significativo a 1 e $5 \%$ de probabilidade. NC: número de condição, obtido no diagnóstico de multicolinearidade; K, constante usada para estabilizar os coeficientes de trilha com multicolinearidade; $\mathrm{R}^{2}$, coeficiente de determinação. 
resultados deste trabalho para as safras e tipos de armazenamento, o que reforça a necessidade de mais pesquisas sobre o assunto.

No experimento I, embora a percentagem de grãos sem defeito não tenha apresentado correlação significativa com a ocorrência de aflatoxinas, verificouse alto efeito direto $(0,54$ e 0,82 , em sacaria e em silo metálico, respectivamente). Este efeito ocorreu principalmente em razão do efeito indireto do conteúdo de cinzas, o que indica que grãos sem defeitos também são acometidos pela toxina, relação também afirmada por Zovico et al. (1999). No experimento II, ocorreu o fato inverso, pois, a correlação foi significativa e de média magnitude, e não houve efeito direto significativo, principalmente em razão dos efeitos indiretos da incidência dos fungos Penicillium sp., Aspergillus sp. e Fusarium sp. A massa volumétrica também não apresentou relação de causa e efeito com a ocorrência de aflatoxinas, nos dois experimentos. Embora, no experimento II, a correlação de Pearson tenha sido significativa e positiva, de magnitude média, em ambas as formas de armazenamento, o efeito direto foi baixo ou negativo, o que ocorreu pela forte influência indireta da incidência de fungos dos três gêneros (Penicillium sp., Aspergillus sp. e Fusarium sp.) e, ainda, do conteúdo de cinzas no armazenamento em sacaria convencional.

A incidência dos fungos dos três gêneros, na maioria dos casos, também não mostrou alta correlação e efeito direto com a ocorrência de aflatoxina. As exceções foram a incidência de Penicillium sp. e de Fusarium sp., no experimento II, em grãos de milho armazenados em sacaria, e a incidência de Aspergillus sp. na safra I, em grãos armazenados em silo metálico, em que a correlação de Pearson e o efeito direto sobre aflatoxinas foram positivos e altos, o que mostra a relação de causa e efeito (Tabela 2). Estas diferenças entre correlações e efeitos nos experimentos I (safra de inverno) e II (safra de verão), quanto à incidência fúngica de Penicillium sp., Aspergillus sp. e Fusarium sp. e a ocorrência de aflatoxinas, podem ser atribuídas às diferentes condições meteorológicas dos períodos de armazenamento, conforme ilustrado na Figura 1, que foram mais propícias à ocorrência de aflatoxinas no armazenamento da safra de verão, pois, a temperatura no início do armazenamento estava acima de $20^{\circ} \mathrm{C}$, e a umidade relativa do ar acima de $68 \%$. Segundo vários autores, estas condições favorecem a germinação e proliferação dos esporos de fungos presentes nos grãos desde a colheita (Marín et al., 1998a; Borém et al., 2006; Almeida et al., 2009; Bento et al., 2012).

Os resultados do presente trabalho corroboram os de Marques et al. (2009), que constataram que a incidência de fungos do gênero Aspergillus foi maior em dois dos três híbridos de milho avaliados na safra de verão 2007/2008 (primavera/verão), em comparação aos grãos dos híbridos de milho avaliados na safrinha 2007 (outono/inverno). A competição entre as espécies de fungos também pode ter interferido nestes resultados. Marín et al. (1998b) descrevem que espécies de Fusarium são capazes de colonizar rapidamente grãos danificados, reduzir a fonte de nutrientes disponível a outros fungos, tais como Aspergillus flavus e interferir no potencial de produção de aflatoxinas. Tédihou et al. (2012) verificaram correlação inversa da incidência de Penicillium sp. com a incidência de A. flavus $(\mathrm{r}=-0,34)$ e a ocorrência de aflatoxina $\mathrm{B}_{1}$ $(\mathrm{r}=-0,21)$. Correlações positivas entre a incidência de A. flavus e as aflatoxinas B1 $(\mathrm{r}=0,54)$ e B2 $(\mathrm{r}=0,52)$ e, também, da incidência de Fusarium sp. com a de $A$. flavus $(\mathrm{r}=0,49)$ e com as aflatoxinas $\mathrm{B}_{1}(\mathrm{r}=0,53)$ e $\mathrm{B}_{2}$ $(r=0,56)$. Bento et al. (2012) encontraram correlação significativa entre a ocorrência de fungos dos gêneros Aspergillus e Penicillium $(\mathrm{r}=0,74)$ em grãos de milho. Os resultados do presente estudo e dos acima citados podem ser explicados pelo fato de os três gêneros de fungos (Aspergillus, Penicillium e Fusarium) serem os mais encontrados como contaminantes em grãos de milho e porque a produção de aflatoxinas é realizada apenas por espécies de Aspergillus, principalmente A. flavus, A. parasiticus e A. nomius (Leeson et al., 1995).

No experimento I, os coeficientes de determinação do modelo de análise de trilha $\left(\mathrm{R}^{2}\right)$ foram $0,66 \mathrm{e}$ 0,62 (com relativos efeitos residuais 0,58 e 0,61 ), enquanto no experimento II foram ambos 0,80 (com efeitos residuais 0,44 ), no armazenamento em sacaria e em silos metálicos, respectivamente. Isto mostra que as variações da variável básica (ocorrência de aflatoxinas) não foram totalmente explicadas pelas nove variáveis explicativas aferidas, e que há outras variáveis influentes na ocorrência de aflatoxinas em grãos de milho armazenados.

Assim, recomenda-se estimar a influência de outras variáveis como: híbridos de milho, época de semeadura, tempo entre a maturidade fisiológica e a colheita, condições meteorológicas de cultivo, tempo de secagem, umidade de colheita e outros.

Pesq. agropec. bras., Brasília, v.50, n.6, p.441-449, jun. 2015 DOI: 10.1590/S0100-204X2015000600002 


\section{Conclusões}

1. Há forte influência das condições ambientais entre safra de inverno e de verão, e entre época e tipo de armazenamento, nos coeficientes de análise de trilha.

2. As variáveis teor de umidade, conteúdo de cinzas, proteínas, lipídios, grãos sem defeitos, massa volumétrica, incidência de Penicillium sp., Aspergillus sp. e Fusarium sp. devem ser consideradas conjuntamente na definição da possibilidade de ocorrência de aflatoxinas na massa de grãos armazenados.

\section{Referências}

ALMEIDA, A.V.A.F.; BOTURA, M.B.; ABREU, R.D.; BITTENCOURT, T.C.C.; BATATINHA, M.J.M. Ocorrência de aflatoxinas em milho destinado à alimentação de aves no Estado da Bahia. Arquivos do Instituto Biológico, v.76, p.353-358, 2009.

BENTO, L.F.; CANEPPELE, M.A.B.; ALBUQUERQUE, M.C. de F. e; KOBAYASTI, L.; CANEPPELE, C.; ANDRADE, P. de J. Ocorrência de fungos e aflatoxinas em grãos de milho. Revista do Instituto Adolfo Lutz, v.71, p.44-49, 2012.

BORÉM, F.M.; RESENDE, O.; MACHADO, J. da C.; FONTENELLE, I.M.R.; SOUSA, F.F. de. Controle de fungos presentes no ar e em sementes de feijão durante armazenamento. Revista Brasileira de Engenharia Agrícola e Ambiental, v.10, p.651-659, 2006. DOI: 10.1590/S1415-43662006000300017.

BRASIL. Mistério da Agricultura, Pecuária e Abastecimento. Portaria $\mathrm{n}^{\circ}$ 845, de 8 de novembro de 1976. [Aprova as especificações para a padronização, classificação e comercialização interna do Milho]. Diário Oficial [da] República Federativa do Brasil, Brasília, 19 nov. 1976.

CARVALHO, F.I.F.; LORENCETTI, C.; BENIN, G. Estimativas e implicações da correlação no melhoramento vegetal. Pelotas: UFPel, 2004. 142p.

COMPANHIA NACIONAL DE ABASTECIMENTO. Acompanhamento da safra brasileira [de] grãos - quinto levantamento - safra 2014/2015. Brasília: Conab, 2015. v.2, n.5, $117 \mathrm{p}$.

CRUZ, C.D. Programa genes: biometria. Viçosa: Ed. da UFV, 2006. 382p.

CRUZ, C.D.; CARNEIRO, P.C.S. Modelos biométricos aplicados ao melhoramento genético. 2.ed. Viçosa: Ed. da UFV, 2006. v.2, $585 \mathrm{p}$.

FARONI, L.R.D.; BARBOSA, G.N. de O.; SARTORI, M.A.; CARDOSO, F. da S.; ALENCAR, E.R. de. Avaliação qualitativa e quantitativa do milho em diferentes condições de armazenamento. Engenharia na Agricultura, v.13, p.193-201, 2005.

HERMANNS, G.; PINTO, F.T.; KITAZAWA, S.E.; NOLL, I.B. Fungos e fumonisinas no período pré-colheita do milho. Ciência e Tecnologia de Alimentos, v.26, p.7-10, 2006. DOI: 10.1590/ S0101-20612006000100002.
LEESON, S.; DIAZ, G.J.; SUMMERS, J.D. Poultry metabolic disorders and mycotoxins. Guelph: University Books, 1995. $352 \mathrm{p}$.

LÚCIO, A.D.; STORCK, L.; KRAUSE, W.; GONÇALVES, R.Q.; NIED, A.H. Relações entre os caracteres de maracujazeiro-azedo. Ciência Rural, v.43, p.225-232, 2013. DOI: 10.1590/S0103-84782013000200006.

MAGAN, N.; ALDRED, D. Post-harvest control strategies: minimizing mycotoxins in the food chain. International Journal of Food Microbiology, v.119, p.131-139, 2007. DOI: 10.1016/j. ijfoodmicro.2007.07.034.

MARÍN, S.; COMPANYS, E.; SANCHIS, V.; RAMOS, A.J.; MAGAN, N. Effect of water activity and temperature on competing abilities of common maize fungi. Mycological Research, v.102, p.959-964, 1998a. DOI: 10.1017/S0953756297005613.

MARÍN, S.; SANCHIS, V.; ARNAU, F.; RAMOS, A.J.; MAGAN, N. Colonisation and competitiveness of Aspergillus and Penicillium species on maize grain in the presence of Fusarium moniliforme and Fusarium proliferatum. International Journal of Food Microbiology, v.45, p.107-117, 1998b. DOI: 10.1016/ S0168-1605(98)00153-6.

MARQUES, O.J.; VIDIGAL FILHO, P.S.; DALPASQUALE, V.A.; SCAPIM, C.A.; PRICINOTTO, L.F.; MACHINSKI JÚNIOR, M. Incidência fúngica e contaminações por micotoxinas em grãos de híbridos comerciais de milho em função da umidade de colheita. Acta Scientiarum. Agronomy, v.31, p.667-675, 2009. DOI: 10.4025 /actasciagron.v31i4.5690.

MAZIERO, M.T.; BERSOT, L. dos S. Micotoxinas em alimentos produzidos no Brasil. Revista Brasileira de Produtos Agroindustriais, v.12, p.89-99, 2010. DOI: 10.15871/1517-8595/ rbpa.v12n1p89-99.

MONTGOMERY, D.C.; PECK, E.A. Introduction to linear regression analysis. New York: J. Wiley, 1981. 504p.

MULUNDA, M.; DZOMA, B.; NYIRENDA, M.; BAKUNZI, F. Mycotoxins occurrence in selected staple food in main markets from Lubumbashi, Democratic Republic of Congo. Journal of Food, Agriculture and Environment, v. 11, p.51-54, 2013.

OLIVEIRA, E.J. de; LIMA, D.S. de; LUCENA, R.S.; MOTTA, T.B.N.; DANTAS, J.L.L. Correlações genéticas e análise de trilha para número de frutos comerciais por planta em mamoeiro. Pesquisa Agropecuária Brasileira, v.45, p.855-862, 2010. DOI: 10.1590/ S0100-204X2010000800011.

ONO, E.Y.S.; BIAZON, L.; SILVA, M. da; VIZONI, E.; SUGIURA, Y.; UENO, Y.; HIROOKA, E.Y. Fumonisins in corn: correlation with Fusarium sp. count, damaged kernels, protein and lipid content. Brazilian Archives of Biology and Technology, v.49, p.63-71, 2006. DOI: 10.1590/S1516-89132006000100008.

PATERSON, R.R.M.; LIMA, N. How will climate change affect mycotoxins in food? Food Research International, v.43, p.1902-1914, 2010. DOI: 10.1016/j.foodres.2009.07.010.

REGRAS para análise de sementes. Brasília: Ministério da Agricultura, Pecuária e Abastecimento, 2009. 395p.

RIOS, S. de A.; BORÉM, A.; GUIMARÃES, P.E. de O.; PAES, M.C.D. Análise de trilha para carotenoides em 
milho. Revista Ceres, v.59, p.368-373, 2012. DOI: 10.1590/ S0034-737X2012000300011.

SALEEMULLAH, K.; IQBAL, A.; KHALIL, I.A.; SHAH, H. Aflatoxin contents of stored and artificially inoculated cereals and nuts. Food Chemistry, v.98, p.699-703, 2006. DOI: 10.1016/j. foodchem.2005.06.034.

SCHUH, G.; GOTTARDI, R.; FERRARI FILHO, E.; ANTUNES, L.E.G.; DIONELLO, R.G. Efeitos de dois métodos de secagem sobre a qualidade físico-química de grãos de milho safrinha-RS, armazenados por 6 meses. Semina: Ciências Agrárias, v.32, p.235-244, 2011. DOI: 10.5433/1679-0359.2011v3 2n1p235.

SINGH, K.; FRISVAD, J.C.; THRANE, U.; MATHUR, S.B. An illustrated manual on identification of some seed-borne Aspergilli, Fusaria, Penicillia and their mycotoxins. Hellerup: Danish Government Institute of Seed Pathology for Developing Countries, 1991. 133p.
TÉDIHOU, E.; OLATINWO, R.; HELL, K.; HAU, B.; HOOGENBOOM, G. Effects of variety, cropping system and soil inoculation with Aspergillus flavus on aflatoxin levels during storage of maize. Tropical Plant Pathology, v.37, p.25-36, 2012. DOI: $10.1590 /$ S1982-56762012000100003.

WRIGHT, S. Correlation and causation. Journal of Agricultural Research, v.20, p.557-585, 1921.

ZENEBON, O.; PASCUET, N.S.; TIGLEA, P. Métodos físico-químicos para análise de alimentos. 4.ed. São Paulo: Instituto Adolfo Lutz, 2008. 1020p.

ZOVICO, C.; FONSECA, H.; CALORI-DOMINGUES, M.A.; GLÓRIA, E.M.; BORGUINI, R.G.; SILVEIRA, V.P.; PIEDADE, S.S.; BARBIN, D. Seleção eletrônica pela cor na descontaminação de amendoim contaminado com aflatoxinas. Scientia Agricola, v.56, p.371-376, 1999. DOI: 10.1590/ S0103-90161999000200016.

Recebido em 15 de julho de 2014 e aprovado em 18 de maio de 2015 